\title{
Celiac Trunk Stenosis Treated by Resection and Splenic Patch Reconstruction - A Case Report and Literature Review
}

\author{
NICOLAE BACALBASA ${ }^{1}$, IRINA BALESCU ${ }^{2}$ and VLADISLAV BRASOVEANU ${ }^{3}$ \\ ${ }^{1}$ Carol Davila University of Medicine and Pharmacy, Bucharest, Romania; \\ ${ }^{2}$ Ponderas Academic Hospital, Bucharest, Romania; \\ ${ }^{3}$ Dan Setlacec Center of Gastrointestinal Disease and Liver Transplantation, \\ Fundeni Clinical Institute, Bucharest, Romania
}

\begin{abstract}
Celiac trunk stenosis is a relatively common finding; the most common causes of this obstruction are median arcuate ligament syndrome, pancreatitis, local invasion of various malignancies originating from the pancreatic body, atherosclerosis or it can be idiopathic. However, most such cases remain asymptomatic for a long period of time, especially due to the presence of a patent collateral circulation originating from the superior mesenteric artery. In certain cases, the patient might become symptomatic, diffuse post-prandial pain being reported. We present the case of a 51-year-old patient diagnosed with celiac axis stenosis who was initially submitted to surgery with the preoperative suspicion of median arcuate ligament syndrome; however, the patient reported the persistence of the abdominal symptoms, leading to suspicion of idiopathic celiac axis stenosis. The patient underwent reoperation, the celiac trunk stenosis being resected, and the continuity of the vascular axis being established using a splenic artery patch. The postoperative course was uneventful, the patient presenting an adequate hepatic blood flow in the first day postoperatively.
\end{abstract}

Celiac trunk stenosis has been reported with an incidence ranging from $12.5-24 \%(1,2)$. The main etiological factors consist of median arcuate ligament compression, followed by arteriosclerosis, pancreatitis, tumoral invasion or congenital abnormalities (3). Moreover, it seems that ethnicity strongly influences the cause of celiac axis stenosis: while in patients from Western countries the main cause is atherosclerosis, in

This article is freely accessible online.

Correspondence to: Irina Balescu, 2A Caraiman Street, Buzau, Romania. Tel: +40 724077709, e-mail: irina.balescu@ponderasah.ro

Key Words: Celiac axis stenosis, median arcuate ligament syndrome, splenic artery patch.
Japanese study groups, the main cause is median arcuate ligament syndrome $(1,3,4)$. According to Sakorafas et al., causes inducing celiac artery stenosis can be classified into three groups: extrinsic (mediate arcuate ligament syndrome, compression induced by the celiac ganglion or surrounding fibrotic transformations), intrinsic (due to arteriosclerosis) and other causes (including congenital malformations, acute or chronic disease, malignant invasion or compression due to chronic pancreatitis) (5).

When it comes to the presence of associated symptoms, it is most often related to the degree of stenosis. With fewer than $1 \%$ of all abdominal arteriograms detecting severe stenosis, it seems that patients with celiac trunk stenosis remain asymptomatic for a long period of time, a reduction of the arterial blood flow by $60-75 \%$ being needed to become symptomatic (6-8). Another important fact which influences the presence of symptomatic disease is the existence of a collateral circulation via the superior mesenteric artery (3). It is estimated that patients presenting celiac artery stenosis will develop collateral circulation via the superior mesenteric artery to the hepatic, gastric and splenic branches (which in a normal patient would be directly supplied by the celiac trunk) $(9,10)$. In patients presenting chronic celiac artery stenosis, adequate collateral circulation develops in up to $80 \%$ of patients; however, in certain cases in which the collateral circulation is not well developed, for various reasons, symptoms might appear.

\section{Case Report}

The 51-year-old patient was initially investigated for diffuse postprandial abdominal pain and weight loss; by angiocomputed tomography he was diagnosed with $70 \%$ stenosis of the celiac trunk extending $8 \mathrm{~mm}$ in length due to an extrinsic compression induced by the median arcuate ligament (Figure 1). The patient was submitted to arcuate ligament resection. However, the symptoms reappeared 6 months later, therefore, another evaluation through angio- 
computed tomography was performed. This time the imaging investigation confirmed persistence of the celiac trunk stenosis (Figure 2); the patient was resubmitted to surgery, resection and reimplantation of the celiac trunk in the abdominal aorta using a splenic artery patch being performed (Figures 3 and 4). The postoperative course was uneventful, the abdominal ultrasound performed on the first postoperative day confirming the presence of a normal blood flow at the level of the patch, as well as at the level of the common hepatic artery, with a resistivity index of 0.71 . The ultrasound revealed a normal aspect of the portal vein, with a mean velocity of $28.6 \mathrm{~cm} / \mathrm{s}$, as well as the absence of any pathological fluid in the abdominal cavity.

\section{Discussion}

Although not frequent conditions, vascular abnormalities or pathological modifications of the main arteries in the upper abdomen (including the celiac trunk and its axes or the superior mesenteric artery) have been widely reported in recent decades, probably due to the improvement of the imagistic studies (11-13). Therefore, various vascular reconstruction techniques have been successfully implemented in order to improve the outcomes of such cases. However, a good understanding of the vascular anatomy is mandatory in order to efficiently diagnose and treat such pathology.

When it comes to the anatomy of the celiac trunk, its origin ranges between the 11th thoracic and the first lumbar vertebra; therefore, a higher origin of this axis is associated with a higher chance of obstruction due to the presence of the median arcuate ligament (3). The median arcuate ligament is a band of connective fibers passing over the abdominal aorta and connecting the diaphragmatic crura. In most cases presenting median arcuate ligament syndrome with celiac axis stenosis, the patient will remain asymptomatic if there are no other associated pathological processes. When symptomatic, these patients will complain of weight loss and epigastric pain after eating (14).

Whenever the cause of stenosis is demonstrated to be an extrinsic one (represented by the presence of an arcuate ligament compression, an enlarged celiac lymph node or a fibro-inflammatory disease), division of the fibrous structures compressing the celiac trunk will provide an efficient method to alleviate the symptoms and, in the meantime, to treat the cause (15). Moreover, in such cases, percutaneous stenting is not recommended due to the higher risk of postprocedural complications, such as thrombosis or stent dislocation, surgery remaining therefore the option of choice (5).

An interesting study regarding celiac axis stenosis was conducted by Ikeda et al., and published in 2009 (3). Among the study population of 990 patients, $2.3 \%$ were diagnosed with stenosis or occlusion of the proximal celiac trunk at a mean age of 58 years. The main causes of proximal celiac axis stenosis were mediate arcuate syndrome in 14 cases followed by direct invasion by a pancreatic body tumor in six cases, and chronic pancreatitis and atherosclerosis in the remnant three cases. In all cases in which median arcuate ligament syndrome was demonstrated, a higher origin of the celiac axis was revealed.

In cases in which celiac axis stenosis is induced by arcuate ligament syndrome and if patent collateral circulation is not present, operative revascularization might be needed. The most cited methods consist of vascular bypass between the post-stenotic area of the celiac trunk and the superior mesenteric artery, or the abdominal aorta directly or by direct arterial re-implantation. In these procedures, grafts used for reconstructions can be prosthetic (such as polytetrafluoroethylene) or autogenous venous or arterial patches (such as the saphenous vein or the splenic artery) $(9,16)$.

Open surgery and vascular reconstructions might be needed if the symptoms persist after sectioning the median arcuate ligament (17). Once the ligamentous fibers are sectioned, a close inspection of the celiac axis is mandatory; according to Reilly et al., persistence of the celiac trunk deformation, the presence of a vascular thrill or of a pressure gradient at this level should lead to vascular reconstruction (18).

The study conducted by Reilly et al., published over three decades ago, included 51 patients diagnosed with celiac axis stenosis due to median arcuate syndrome ligament, while the main surgical procedures performed included celiac axis decompression in 16 cases, celiac axis decompression followed by dilatation in 17 cases, and celiac decompression followed by reconstruction through direct reimplantation of graft interposition in 18 patients. The long-term follow-up revealed a positive outcome (defined by the absence of recurrent symptoms) in $53 \%$ of cases treated by celiac decompression alone, and in $76 \%$ of cases treated by decompression and revascularization. In conclusion, the authors underlined the possibility of the coexistence of multiple factors inducing this syndrome, a simple decompression not being always enough to achieve a good control of the symptoms (18).

In a more recent study conducted by Grotemeyer et al. published in 2009, the authors reported a group of 18 patients diagnosed with median arcuate ligament syndrome and treated by open surgery. Although in all cases a decompression was performed, a degree of vascular reconstruction was needed in 11 cases; therefore, a vein interposition between the aorta and the celiac trunk was performed in six cases, aorto-hepatic vein interposition was needed in one case, resection of the stenosis and end-to-end anastomosis was performed in two cases, venous celiac arterioplasty was performed in one case, while transaortic stent removal was needed in another patient. In all cases, the venous patch originated from the greater saphenous vein. Among cases in which an aorto-celiac vein interposition was performed, it is interesting to note that in two cases, the 


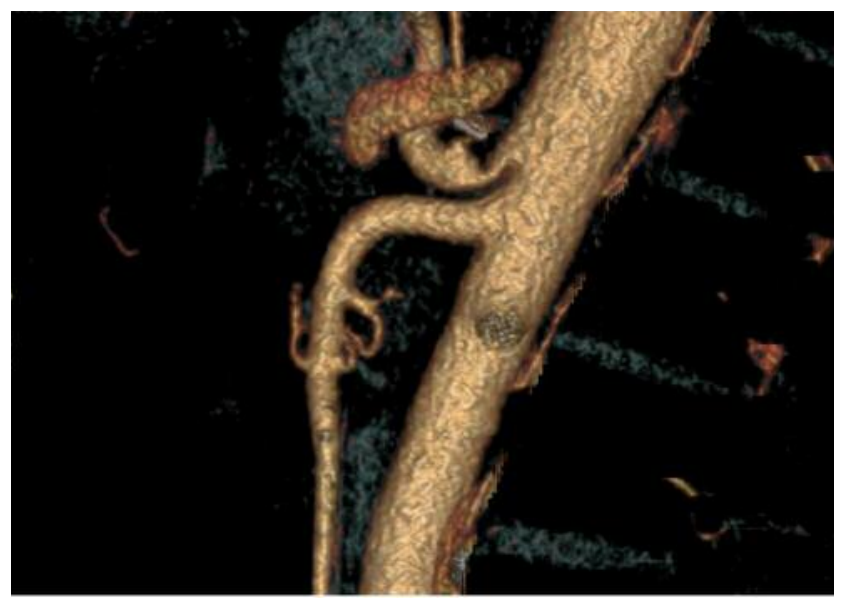

Figure 1. Initial aspect of the angio-computed tomography revealing a celiac axis stenosis.

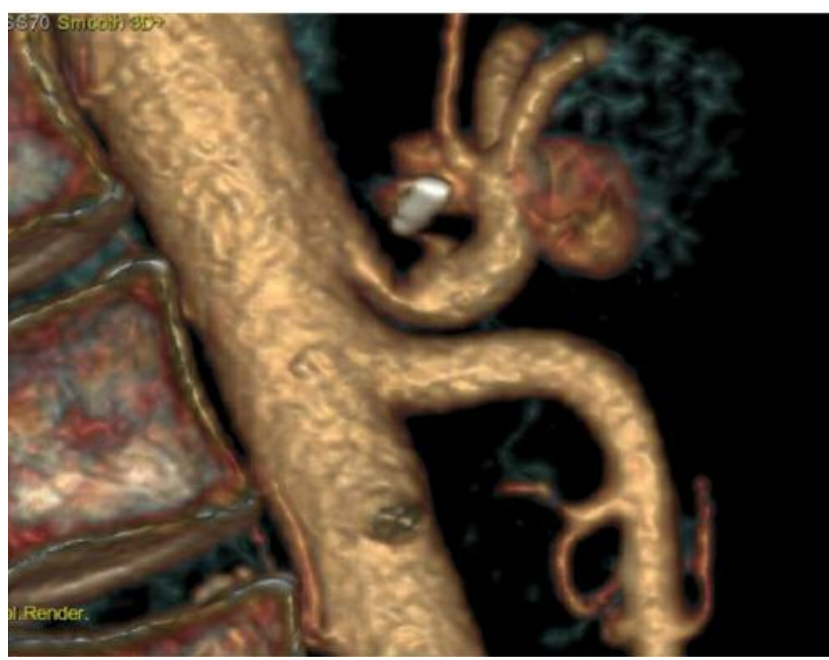

Figure 2. The aspect 6 months after sectioning the fibers of the arcuate ligament, demonstrating the persistence of the celiac trunk stenosis.

reconstruction was performed as a second stage procedure; the two patients had been initially submitted only to celiac trunk decompression, and due to the persistence of the symptoms they underwent reoperation on the eighth and 14th postoperative day, respectively. Another interesting case reported in this series was that of a 20 -year-old patient who redeveloped symptoms 3 months after initial surgery and in whom a transposition of the splenic artery in the supravisceral aorta was performed. After a mean follow-up period of 3.5 years, 11 patients were completely asymptomatic; however, among these cases, only six had been initially submitted only to decompression, in all the other cases, a degree of reconstructive surgery was also associated (19).

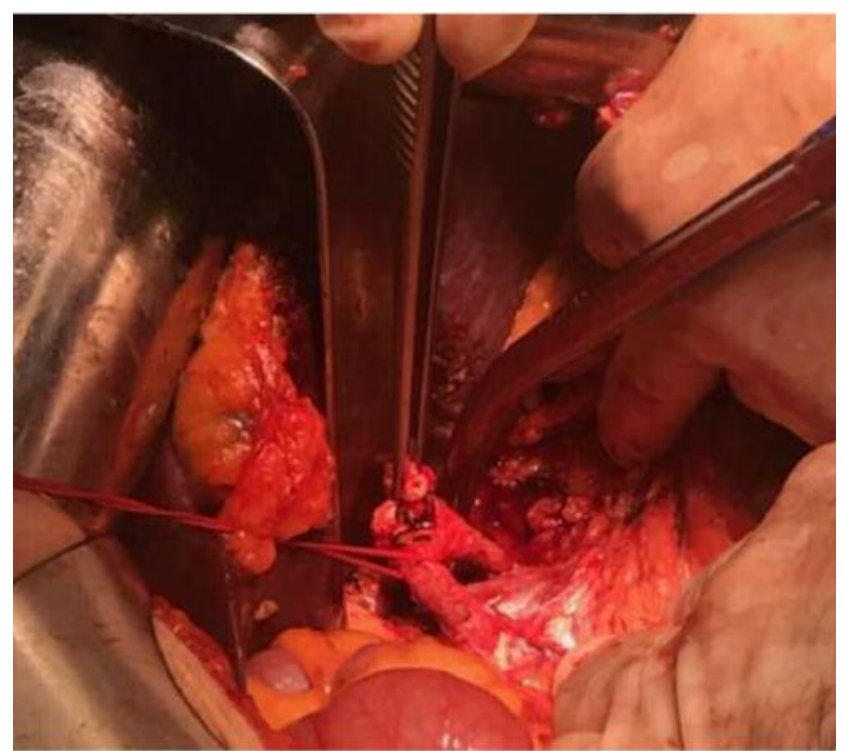

Figure 3. Intraoperative aspect after vascular dissection of the celiac trunk and its branches.

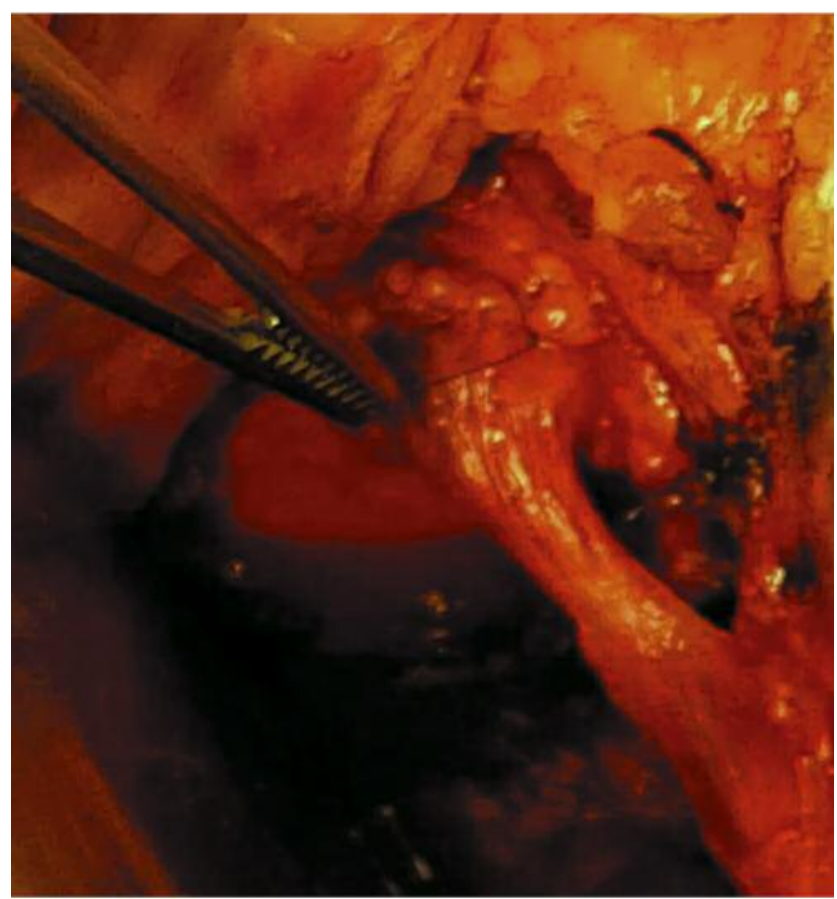

Figure 4. The final aspect after reconstruction of the celiac trunk using a splenic artery patch.

Another interesting study comes from Duran et al. and was published in 2017; the study included 31 patients diagnosed with median arcuate ligament syndrome treated by decompression (in 17 cases) or decompression and 
reconstruction (in 14 cases) over a period of 20 years. Among cases necessitating vascular reconstruction, the splenic artery was used in two patients. The overall morbidity rate was $16.1 \%$, revisional surgery of the reconstructive procedure being needed in four cases (none of them being initially submitted to a patch from the splenic artery). After a median follow-up period of 12, 24 and 60 months, $93.3 \%, 77.8 \%$ and $69.1 \%$ of the patients submitted to decompression and $100 \%, 83.3 \%$ and $83.3 \%$ of the patients submitted to reconstruction, respectively, were asymptomatic; however, this difference was not statistically significant $(p=0.72)(20)$.

\section{Conclusion}

Open treatment of median arcuate ligament syndrome through fibrous transsection might be followed in certain cases by the persistence of the celiac artery syndrome (translated through the persistence or reaparition of the symptomatology). In such cases reconstructive surgery might be needed in order to alleviate the symptoms. Depending on the anatomical particularities of each patient, the procedures of choice might consist of venous patch (usually from the greater saphenous vein), arterial patch (using the splenic artery), or stent placement. Although not frequently reported, splenic artery patch seems to be a safe and efficient method, with good results in terms of late follow-up results.

\section{References}

1 Reuter SR and Olin T: Stenosis of the celiac artery. Radiology 85: 616-627, 1965.

2 Derrick JR, Pollard HS and MooreORM: The pattern of arteriosclerotic narrowing of the celiac and superior mesenteric arteries. Ann Surg 149: 684-689, 1959.

3 Ikeda O, Tamura Y, Nakasone Y and Yamashita Y: Celiac artery stenosis/occlusion treated by interventional radiology. Eur $\mathbf{J}$ Radiol 71: 369-377, 2009.

4 Loffeld RJ, Overtoom HA and Rauwerda JA: The celiac axis compression syndrome. Report of 5 cases. Digestion 56: 534$537,1995$.

5 Sakorafas GH, Sarr MG and Peros G: Celiac artery stenosis: an underappreciated and unpleasant surprise in patients undergoing pancreaticoduodenectomy. J Am Coll Surg 206: 349-356, 2008.

6 Cornell SH: Severe stenosis of the celiac artery. Analysis of patients with and without symptoms. Radiology 99: 311-316, 1971.

7 Kopecky KK, Stine SB, Dalsing MC and Gottlieb K: Median arcuate ligament syndrome with multivessel involvement: diagnosis with spiral CT angiography. Abdom Imaging 22: 318320, 1997.
8 Winter TC, III, Nghiem HV, Freeny PC, Hommeyer SC and Mack LA: Hepatic arterial anatomy: demonstration of normal supply and vascular variants with three-dimensional CT angiography. Radiographics 15: 771-780, 1995.

9 Thompson NW, Eckhauser FE, Talpos $G$ and Cho KJ: Pancreaticoduodenectomy and celiac occlusive disease. Ann Surg 193: 399-406, 1981.

10 Berney T, Pretre R, Chassot G and Morel P: The role of revascularization in celiac occlusion and pancreatoduodenectomy. Am J Surg 176: 352-356, 1998.

11 Brasoveanu V, Anghel C, Barbu I, Pautov M, Ionescu MI, Motthor M, Balescu I, Dima $\mathrm{S}$ and Bacalbasa $\mathrm{N}$ : Pancreatoduodenectomy en bloc with portal and superior mesenteric artery resection - a case report and literature review. Anticancer Res 35: 1613-1618, 2015.

12 Bacalbasa N, Brezean I, Anghel C, Barbu I, Pautov M, Balescu $\mathrm{I}$ and Brasoveanu V: Management of a fulminant upper gastrointestinal bleeding exteriorized through hemobilia due to arteriobiliary fistula between the common bile duct and a right hepatic artery aneurysm - a case report. In Vivo 31: 983-989, 2017.

13 Bacalbasa N, Brezean I, Anghel C, Barbu I, Pautov M, Balescu I and Brasoveanu V: Successful resection and vascular ligation of a large hepatic artery aneurysm - a case report and literature review. In Vivo 31: 979-982, 2017.

14 Horton KM, Talamini MA and Fishman EK: Median arcuate ligament syndrome: evaluation with $\mathrm{CT}$ angiography. Radiographics 25: 1177-1182, 2005.

15 van Wanroij JL, van Petersen AS, Huisman AB, Mensink PB, Gerrits DG, Kolkman JJ and Geelkerken RH: Endovascular treatment of chronic splanchnic syndrome. Eur J Vasc Endovasc Surg 28: 193-200, 2004.

16 Miyata M, Takao T, Okuda A, Sasako Y and Sunada S: Pancreatoduodenectomy for periampullary cancer associated with celiac occlusion: a case report. Surgery 103: 261-263, 1988.

17 Duncan AA: Median arcuate ligament syndrome. Curr Treat Options Cardiovasc Med 10: 112-116, 2008.

18 Reilly LM, Ammar AD, Stoney RJ and Ehrenfeld WK: Late results following operative repair for celiac artery compression syndrome. J Vasc Surg 2: 79-91, 1985.

19 Grotemeyer D, Duran M, Iskandar F, Blondin D, Nguyen K and Sandmann W: Median arcuate ligament syndrome: vascular surgical therapy and follow-up of 18 patients. Langenbecks Arch Surg 394: 1085-1092, 2009.

20 Duran M, Simon F, Ertas N, Schelzig H and Floros N: Open vascular treatment of median arcuate ligament syndrome. BMC Surg 17: 95, 2017. 